Communications in Contemporary Mathematics

Vol. 9, No. 6 (2007) 941-942

(C) World Scientific Publishing Company

\title{
Author Index Volume 9 (2007)
}

Akaho, M., Morse homology and manifolds with boundary

Alon, N., Benjamini, I., Lubetzky, E. \& Sodin, S., Non-backtracking random walks mix faster

Barron, K., The moduli space of $N=2$ superRiemann spheres with tubes

Bartolucci, D., Leoni, F. \& Orsina, L., Uniform estimates and blow-up analysis for the Emden exponential equation in any dimension

Benjamini, I., see Alon, N.

Branson, T. \& Gover, A. R., Pontrjagin forms and invariant objects related to the Q-curvature

Bruzzo, U. \& Graña Otero, B., Numerically flat Higgs vector bundles

Cao, J. \& Tang, H., An intrinsic proof of Gromoll-Grove diameter rigidity theorem

Chiron, D., On the definitions of Sobolev and $B V$ spaces into singular spaces and the trace problem

Cook, W. J., Li, H. \& Misra, K. C., A recurrence relation for characters of highest weight integrable modules for affine Lie algebras

Dávila, J. \& Dupaigne, L., Perturbing singular solutions of the Gelfand problem
9 (2007) 301 nential decay of the solutions for nonlinear biharmonic equations

9 (2007) 585

9 (2007) 857

9 (2007) 163

9 (2007) 585

9 (2007) 335

9 (2007) 437

9 (2007) 401

9 (2007) 473

9 (2007) 121

9 (2007) 639

Ding, F. \& Geiges, H., Legendrian knots and links classified by classical invariants

Ding, K. \& Wu, S., Inversions in classical Weyl groups

Dupaigne, L., see Dávila, J.

Eidelman, S. D. \& Eidelman, Y., On the regularity of the extremal solution of some Dirichlet problem

Eidelman, Y., see Eidelman, S. D.

Fialowski, A. \& Penkava, M., Deformations of four-dimensional Lie algebras

García-Melián, J., Rossi, J. D. \& Suárez, A., The competition between incoming and outgoing fluxes in an elliptic problem

Geiges, H., see Ding, F.

Gover, A. R., see Branson, $\mathrm{T}$.

Graña Otero, B., see Bruzzo, U.

Grünfeld, C. P., A nonlinear evolution equation in an ordered space, arising from kinetic theory

Habets, P. \& Omari, P., Multiple positive solutions of a one-dimensional prescribed mean curvature problem
Deng, Y. \& Li, Y., Expo-

9 (2007) 753

9 (2007) 31

9 (2007) 135

9 (2007) 1

9 (2007) 639

9 (2007) 31
9 (2007) 217

9 (2007) 41

9 (2007) 781

9 (2007) 135

9 (2007) 335

9 (2007) 437

9 (2007) 701 
Hang, F., On the higher order conformal covariant operators on the sphere

Ignat, R. \& Poliakovsky, A., On the relation between minimizers of a $\Gamma$-limit energy and optimal lifting in $B V$-space

Kawohl, B. \& Schuricht, F., Dirichlet problems for the 1-Laplace operator, including the eigenvalue problem

Leoni, F., see Bartolucci, D.

Li, H., see Cook, W. J.

Li, H., A smash product construction of nonlocal vertex algebras

Li, Y., see Deng, Y.

Lim, Y., Defining an $S U(3)-$ Casson / $U(2)$-SeibergWitten integer invariantfor integral homology 3-spheres

Lubetzky, E., see Alon, N.

Machihara, S., Dirac equation with certain quadratic nonlinearities in one space dimension

Misra, K. C., see Cook, W. J.

Omari, P., see Habets, P.

Orsina, L., see Bartolucci, D.

Park, J. \& Yun, K.-H., Rational blow-downs and nonsymplectic 4manifolds with one basic class

Penkava, M., see Fialowski, A.

Poliakovsky, A., see Ignat, R.

Rossi, J. D., see GarcíaMelián, J.

Ruan, W.-D., Generalized special Lagrangian torus fibration for Calabi-Yau hypersurfaces in toric varieties I
9 (2007) 279

9 (2007) 447

9 (2007) 515

9 (2007) 163

9 (2007) 121

9 (2007) 605

9 (2007) 753

9 (2007) 359

9 (2007) 585

9 (2007) 421

9 (2007) 121

9 (2007) 701

9 (2007) 163

9 (2007) 681

9 (2007) 41

9 (2007) 447

9 (2007) 781

9 (2007) 201
Rudyak, Yu. B. \& Schlenk, F., Minimal atlases of closed symplectic manifolds

Schlenk, F., see Rudyak, Yu. B.

Schuricht, F., see Kawohl, B.

Simon, G. B., The nonlinear Maslov index and the Calabi homomorphism

Snoussi, S. \& Tayachi, S., Asymptotically selfsimilar global solutions of a damped wave equation

Sodin, S., see Alon, N.

$\mathrm{Su}$, J., Wang, Z.-Q. \& Willem, M., Nonlinear Schrödinger equations with unbounded and decaying radial potentials

Suárez, A., see GarcíaMelián, J.

Suh, Y. J. \& Yang, H. Y., The scalar curvature of minimal hypersurfaces in a unit sphere

9 (2007) 811

9 (2007) 811

9 (2007) 515

9 (2007) 769

9 (2007) 253

9 (2007) 585

9 (2007) 571

9 (2007) 781

Tang, H., see Cao, J.

Tayachi, S., see Snoussi, S.

Vázquez, J. L., Porous medium flow in a tube. Traveling waves and KPP behavior

Wang, Z.-Q., see Su, J.

Willem, M., see $\mathrm{Su}, \mathrm{J}$.

Wu, S., see Ding, K.

Xavier, F., Rigidity of the identity

Yan, Y., Some compactness results related to scalar curvature deformation

Yang, H. Y., see Suh, Y. J.

$\mathrm{Yu}, \mathrm{Z}$., On the study of one flow for ASD connection

Yun, K.-H., see Park, J.

Zhang, K., On the principle of controlled $L^{\infty}$ convergence implies almost everywhere convergence for gradients
9 (2007) 183

9 (2007) 401

9 (2007) 253

9 (2007) 731

9 (2007) 571

9 (2007) 571

9 (2007) 1

9 (2007) 691

9 (2007) 81

9 (2007) 183

9 (2007) 545

9 (2007) 681

9 (2007) 21 